11

\title{
Способ слепого обнаружения сверхширокополосных хаотических радиоимпульсов на фоне межимпульсной интерференции
}

\author{
() Л.В. Кузьмин ${ }^{1,2}$, А.В. Гриневич ${ }^{3}$ \\ ${ }^{1}$ Институт радиотехники и электроники им. В.А. Котельникова РАН, Москва, Россия \\ ${ }^{2}$ Московский физико-технический институт (Национальный исследовательский университет), Долгопрудный, \\ Московская обл., Россия \\ ${ }^{3}$ Московский государственный технический университет им. Н.Э. Баумана, Москва, Россия \\ E-mail: Ivkuzmin@gmail.com
}

Поступило в Редакцию 16 апреля 2019г.

В окончательной редакции 6 мая 2019г.

Принято к публикации 6 мая 2019г.

Решается задача слепого обнаружения сверхширокополосных хаотических радиоимпульсов сверхвысокочастотного диапазона, прошедших через канал с многолучевым распространением. На основе результатов измерений распространения сверхширокополосных хаотических радиоимпульсов в реальном беспроводном канале предлагается метод их обнаружения, не подверженный влиянию межимпульсной интерференции.

Ключевые слова: сверхширокополосные сигналы, хаотические сигналы, хаотические радиоимпульсы, многолучевое распространение, слепое обнаружение, многолучевое замирание.

DOI: 10.21883/PJTF.2019.16.48154.17840

Предлагается метод слепого энергетического обнаружения сверхширокополосных (СШП) хаотических радиоимпульсов СВЧ-диапазона, нивелирующий влияние межимпульсной интерференции из-за многолучевого распространения СШП-сигнала [1] в системах беспроводной передачи информации СВЧ-диапазона малого радиуса действия: в жилых и промышленных помещениях, в условиях загородной застройки. Метод является слепым, так как не требует априорных измерений параметров распространения СШП-сигнала в условиях конкретного многолучевого канала.

Некогерентное обнаружение хаотических радиоимпульсов не представляет проблемы в многолучевом канале, если задержки в распространении отраженных лучей не превосходят длительности межимпульсных защитных интервалов [2-4]. В этом случае межимпульсной интерференции нет, поэтому характеристики энергетического обнаружения хаотических радиоимпульсов в многолучевом канале по вероятности ошибки близки к характеристикам в канале с одним лучом и гауссовским шумом $[2,3]$.

Проблемы с когерентным и некогерентным обнаружением СШП-сигналов появляются как при использовании хаотических СШП-радиоимпульсов [5,6], так и при использовании ультракоротких СШП-импульсов [7], когда задержки в приходе отраженных лучей непредсказуемо выходят за пределы межимпульсных защитных интервалов, что типично, например, для мобильных беспроводных систем. Для решения этой проблемы предложены и исследованы энергетические методы обнаружения при помощи априорного статистического оценивания параметров многолучевого СШП-канала [5,7], слепые энергетические [8-12] и RAKE-методы (см. работы $[13,14]$ и ссылки в них) для ультракоротких импульсов. В случае сверхширокополосных RAKE-приемников рассматривались способы выбора лучей на базе схем PRAKE (partial RAKE), SRAKE (selective RAKE) [13] и их модификации [14]. Было установлено, что теоретические характеристики таких RAKE-приемников, как минимум, не превосходят по вероятности ошибок обнаружения характеристик энергетических приемников, но уступают им по простоте реализации. Связано это с большим числом лучей в СШП-канале, которые могут иметь приблизительно одну и ту же мощность $[14,15]$, и прием СШП-сигналов необходимо осуществлять по всем таким лучам.

Здесь предлагается подход к обнаружению последовательности хаотических СШП-радиоимпульсов на фоне межимпульсной интерференции в рамках слепого энергетического обнаружения. Метод опирается на естественное свойство хаотического СШП-сигнала некогерентное сложение в точке приема всех лучей, попадающих в приемник, что снимает проблему выделения наиболее мощных лучей, характерную для RAKE-приемника. Метод анализируется на базе экспериментальных данных по распространению таких импульсов длительностью $T_{P}=83 \mathrm{~ns}$ с периодом их следования $T=166 \mathrm{~ns}$ (т.е. импульсы разделены паузами длительности $\left.T-T_{P}=83 \mathrm{~ns}\right)$ через беспроводный канал.

Предполагается, что мощность помехи от межимпульсной интерференции больше мощности теплового шума приемника, поэтому последним можно пренебречь. Физически это оправдано, так как в противном случае при условии ограниченной чувствительности любого реального приемника он не будет способен обнаруживать многолучевой сигнал на фоне теплового 


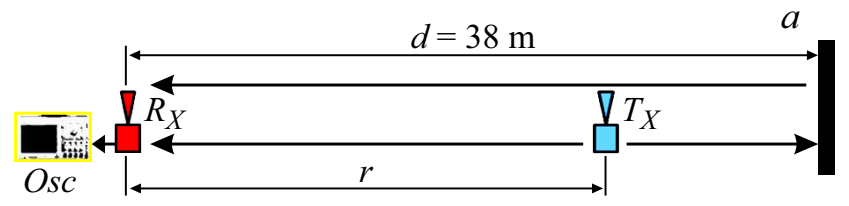

$b$

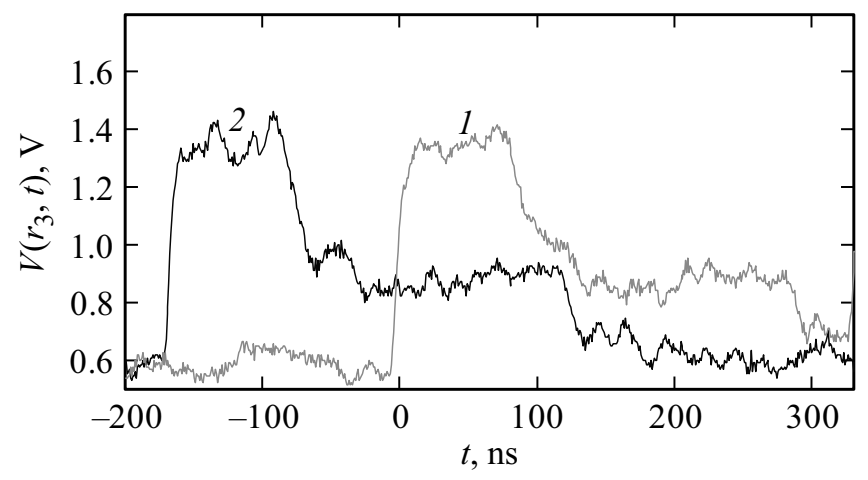

Рис. 1. $a-$ схема проведения измерений: $R_{X}-$ приемник, $T_{X}$ - излучатель, $O s c$ - запоминающий осциллограф, $d=38 \mathrm{~m}, r$ меняется в диапазоне от 2 до $32 \mathrm{~m} ; b-$ фрагменты экспериментальных реализаций огибающих хаотических радиоимпульсов $V\left(r_{3}, t\right)$ для $r_{3}=8 \mathrm{~m}$, иллюстрирующие межимпульсную интерференцию в приемнике: 1 - огибающая текущего импульса, подлежащего обнаружению, 2 - огибающая предшествующего импульса.

шума [6], и поставленную задачу рассматривать станет бессмысленно.

В ходе эксперимента по схеме, показанной на рис. 1, $a$, межимпульсная интерференция наблюдалась в точке приема $R_{X}$ в реальном беспроводном канале, где приемник $R_{X}$ был неподвижен, а излучатель $T_{X}$ перемещался так, что расстояние между приемником и излучателем составляло $r_{1}=32 \mathrm{~m}, r_{2}=16 \mathrm{~m}, r_{3}=8 \mathrm{~m}, r_{4}=4 \mathrm{~m}$ и $r_{5}=2 \mathrm{~m}$.

Как показано в [6], такая схема измерений создает необходимые для возникновения межимпульсной интерференции условия и позволяет в эксперименте наблюдать два луча: прямой и отраженный от массивной металлической двери, расположенной в торце коридора здания ИРЭ им. В.А. Котельникова РАН, где проводился эксперимент [6]. Излучатель $T_{X}$ формировал последовательность хаотических радиоимпульсов, приемник $R_{X}$ формировал их огибающую. Запоминающий осциллограф фиксировал реализации огибающих с частотой выборки $2.5 \mathrm{GHz}$. На рис. $1, b$ проиллюстрировано возникновение межимпульсной интерференции на примере двух реализаций огибающих, выбранных из $N_{3} \approx 100$ огибающих импульсов $V_{j}\left(r_{3}, t\right)\left(j=1, \ldots, N_{3}\right)$, зафиксированных для расстояния $r_{3}=8 \mathrm{~m}$ между излучателем и приемником в ситуации, когда в приемник последовательно поступило два хаотических радиоимпульса, разделенных межимпульсным интервалом. Межимпульсная интерференция проявляется наложением на позицию текущего импульса (кривая 1), подлежащего обнаружению, сигнала огибающей от предшествующего импульса (кривая 2).

Приемник $R_{X}$ состоял из логарифмического детектоpa [15] и малошумящего усилителя с коэффициентом усиления $10^{2}$. Приемник в своей рабочей зоне преобразует входную мощность от $3 \cdot 10^{-9}$ до $\sim 1 \mathrm{~mW}$ в выходное напряжение из диапазона от 0.5 до $2 \mathrm{~V}$ по логарифмическому закону

$$
V(r, t)=10 \alpha \lg \left(P(r, t) / P_{0}\right),
$$

где $P_{0}=1 \mathrm{~mW} ; \alpha=0.021 \mathrm{~V} / \mathrm{dB}$ - крутизна характеристики логарифмического детектора; $P(r, t)$ - мощность сигнала, поступающая в приемник от излучателя, находящегося на расстоянии $r$ от него. Благодаря логарифмическому закону преобразования мощности в напряжение амплитуда огибающей сигнала на выходе приемника меняется по линейному закону, в то время как мощность сигнала на входе может меняться на несколько порядков по степенному закону.

При помощи такого приемника в канале с одним лучом реализуется метод обнаружения, который не требует априорного определения порога принятия решения $H$ о поступлении импульса или об его отсутствии [4]. Пороговое значение мощности $H$, превышение которого сигнализирует о поступлении импульса, является фиксированным и определяется суммарной мощностью теплового шума приемника и мощностью помехового сигнала, возникающего из-за погрешностей технической реализации.

Здесь задача создания слепого правила принятия решения о поступлении или отсутствии импульсов на фоне межимпульсной интерференции анализируется в ситуации, когда в приемник поступает сигнал импульс-паузаотсутствие импульса. В этом случае имеется ненулевая вероятность принять отсутствие импульса после паузы за приход импульса, если пороговое значение мощности $H$ выбирать так, как это делается в канале с одним лучом. В ситуациях импульс-паузаимпульс, отсутствие импульса-пауза-импульс и отсутствие импульса-пауза-отсутствие импульса ошибочного обнаружения при использовании фиксированного порога $H$ не происходит.

Для предотвращения ошибочных обнаружений предлагается использовать знание о закономерности затухания многолучевого сигнала, который перед попаданием в приемник проходит больший путь, чем прямой луч, поэтому его мощность, во-первых, заведомо меньше, чем мощность прямого луча, а во-вторых, для этой мощности можно получить оценку сверху, зная периодичность следования импульсов, показатель затухания сигнала в канале и исходя из некогерентного сложения лучей в приемнике благодаря малому времени когерентности хаотического сигнала [6]. Указанные параметры не зависят от условий распространения сигнала в каждом конкретном случае, поэтому предлагаемый метод является слепым. 

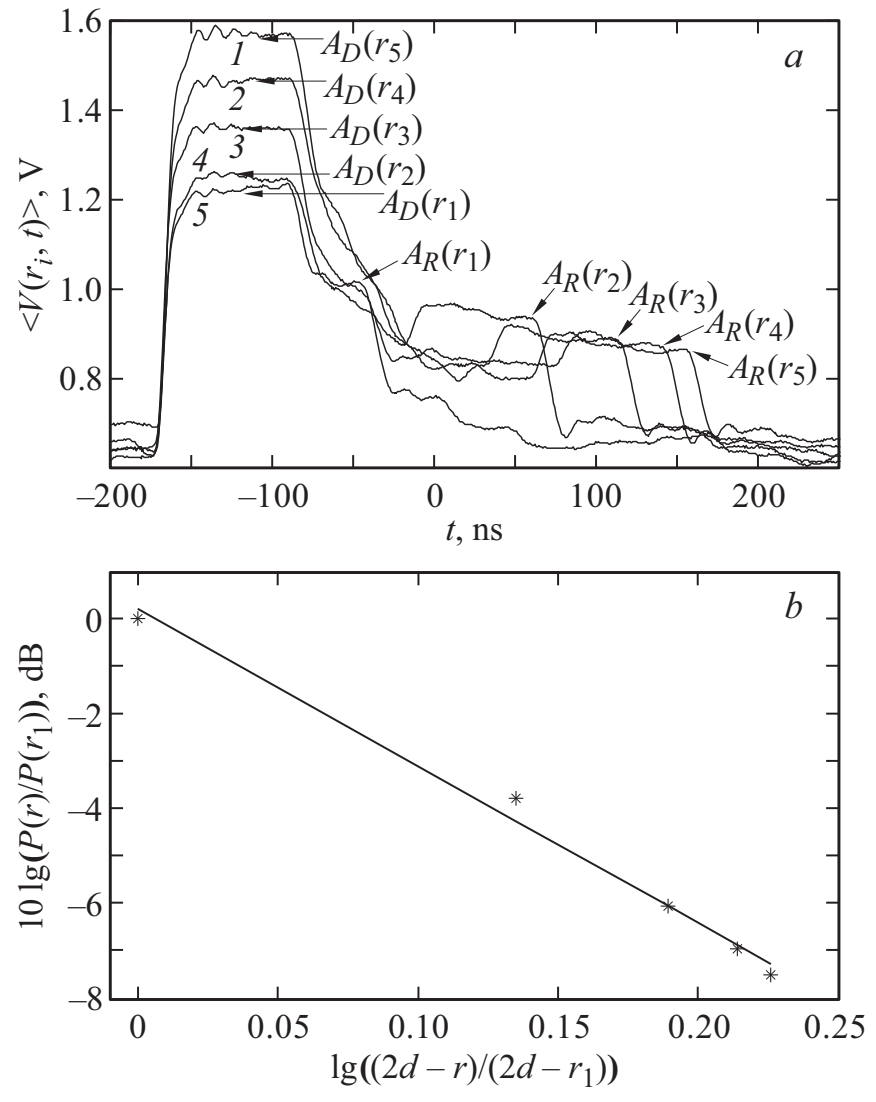

Рис. 2. $a-$ усредненные формы огибающей импульсов $\left\langle V\left(r_{i}, t\right)\right\rangle$, на которых отмечены средние амплитуды $\left\langle A_{D}\left(r_{i}\right)\right\rangle$ прямого и $\left\langle A_{R}\left(r_{i}\right)\right\rangle$ отраженных лучей, фиксируемых приемником на расстоянии $r_{i}(\mathrm{~m})$ от излучателя для импульсов мощностью $100 \mathrm{~mW}:\left\langle A_{R}\left(r_{5}=2\right)\right\rangle=0.85 \mathrm{~V} \quad(1)$, $\left\langle A_{R}\left(r_{4}=4\right)\right\rangle=0.86 \mathrm{~V} \quad(2), \quad\left\langle A_{R}\left(r_{3}=8\right)\right\rangle=0.88 \mathrm{~V} \quad(3)$, $\left\langle A_{R}\left(r_{2}=16\right)\right\rangle=0.93 \mathrm{~V} \quad(4),\left\langle A_{R}\left(r_{1}=32\right)\right\rangle=1.01 \mathrm{~V} \quad(5) ; b-$ построенная по этим данным зависимость отношения мощностей $P(r) / P\left(r_{1}\right)$ для отраженного луча (в децибелах) от отношения расстояний $\lg \left((2 d-r) /\left(2 d-r_{1}\right)\right)$ между излучателем и приемником: точки - результаты измерений, сплошная линия - аппроксимация.

В беспроводном канале мощность $P_{R}$ сигнала в точке приема пропорциональна $P_{T} / r^{n}[1]$, где $n=n_{0}=2$ для свободного пространства, $n=n_{1}<2$ в многолучевом канале, когда имеется прямой луч. В отсутствие прямого луча можно ожидать, что $n=n_{2}>2$; более того, $n=n_{2} \geqslant 2 n_{1}$, так как речь идет о переотраженном сигнале.

Здесь показатель затухания переотраженного сигнала был определен экспериментально по аналогии с [6], где был измерен показатель затухания для прямого луча, который оказался равен $n_{1} \approx 1.5$. Для экспериментального определения значения показателя затухания переотраженных лучей по методике [6] вычислялась усредненная форма огибающей импульсов как

$$
\left\langle V\left(r_{i}, t\right)\right\rangle=\left(1 / N_{i}\right) \sum_{j=1}^{N_{i}} V_{j}\left(r_{i}, t\right) .
$$

Полученные усредненные формы $\left\langle V\left(r_{i}, t\right)\right\rangle$ для импульсов с излучаемой мощностью $100 \mathrm{~mW}$ для $r_{5}=2 \mathrm{~m}$, $r_{4}=4 \mathrm{~m}, r_{3}=8 \mathrm{~m}, r_{2}=16 \mathrm{~m}$ и $r_{1}=32 \mathrm{~m}$ изображены на рис. $2, a$. Верхняя кривая 1 на рис. $2, a$ соответствует минимальному расстоянию между излучателем и приемником, нижняя (кривая 5) - максимальному. По усредненным огибающим $\left\langle V\left(r_{i}, t\right)\right\rangle$ были определены средние амплитуды импульсов $\left\langle A_{R}\left(r_{i}\right)\right\rangle$ отраженного луча. Показатель затухания мощности сигнала с расстоянием для отраженных лучей был рассчитан при помощи (1) через разность между амплитудой импульса для расстояния $r_{i}$ и для расстояния $r_{1}:\left\langle A_{R}\left(r_{i}\right)\right\rangle-\left\langle A_{R}\left(r_{1}\right)\right\rangle=10 \alpha \lg \left(P_{R}\left(r_{1}\right) / P_{R}\left(r_{i}\right)\right)$. Поскольку отношение мощности отраженного луча $P_{R}\left(r_{1}\right)$ для расстояния $r_{1}$ между излучателем $T_{X}$ и приемником $R_{X}$ к мощности $P_{R}\left(r_{i}\right)$ для расстоянии $r_{i}$ составляет $P_{R}\left(r_{1}\right) / P_{R}\left(r_{i}\right)=\left(\left(2 d-r_{i}\right) /\left(2 d-r_{1}\right)\right)^{n_{2}}$, имеем

$$
\left(\left\langle A\left(r_{i}\right)\right\rangle-\left\langle A\left(r_{1}\right)\right\rangle\right) / \alpha=10 n_{2} \lg \left(\left(2 d-r_{i}\right) /\left(2 d-r_{1}\right)\right) .
$$

По экспериментально измеренной зависимости $\left(\left\langle A\left(r_{i}\right)\right\rangle-\left\langle A\left(r_{1}\right)\right\rangle\right) \quad$ от $\lg \left(\left(2 d-r_{i}\right) /\left(2 d-r_{1}\right)\right) \quad$ был определен показатель затухания $n_{2}$ путем определения наклона прямой (2), аппроксимирующей экспериментальные данные (рис. 2,b). Показатель затухания оказался равен $n_{2}=3.3$ для отраженного луча, что согласуется с соотношением $n_{2} \geqslant 2 n_{1}, n_{1} \approx 1.5$.

Опираясь на закономерность падения мощности отраженного луча в зависимости от длины его пробега, можно вычислить пороговое значение мощности $H$, выше которого приемником будет обнаруживаться только прямой луч и которого заведомо не превысит отраженный луч, т.е. реализовать слепое обнаружение хаотических радиоимпульсов по прямому лучу.

Расчет порога $H$ осуществляется на основе периода следования импульсов $T$ и показателей затухания прямо-

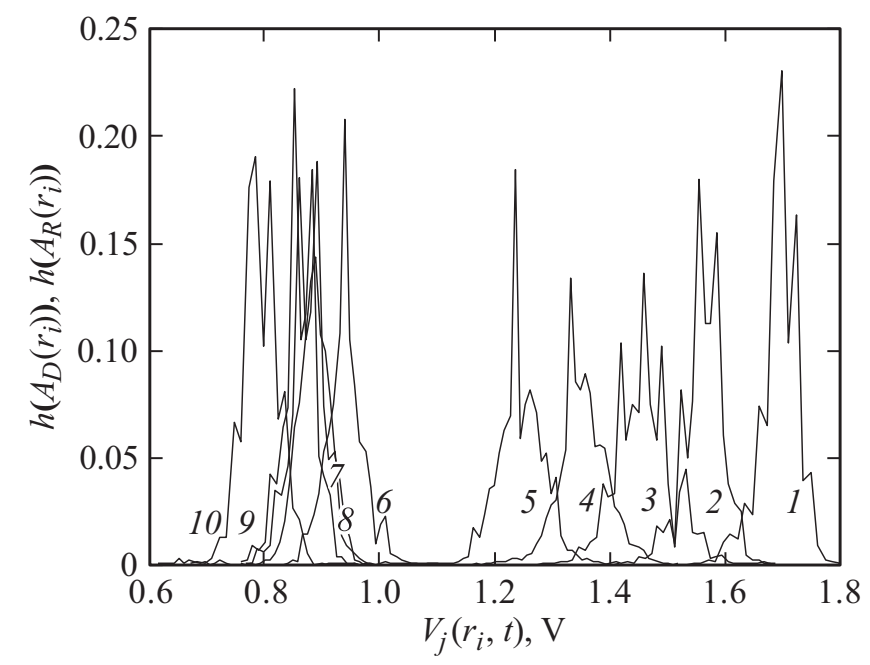

Рис. 3. Распределения амплитуд импульсов прямого $h\left(A_{D}\left(r_{i}\right)\right)$ $\left(1-r_{1}=32 \mathrm{~m}, 2-r_{2}=16 \mathrm{~m}, 3-r_{3}=8 \mathrm{~m}, 4-r_{4}=4 \mathrm{~m}\right.$, $\left.5-r_{5}=2 \mathrm{~m}\right)$ и отраженного лучей $h\left(A_{R}\left(r_{i}\right)\right)\left(6-r_{5}=2 \mathrm{~m}\right.$, $\left.7-r_{4}=4 \mathrm{~m}, 8-r_{3}=8 \mathrm{~m}, 9-r_{2}=16 \mathrm{~m}, 10-r_{1}=32 \mathrm{~m}\right)$. 
го $n_{1}$ и отраженного $n_{2}$ лучей. Так как мощность прямого луча в приемнике составляет

$$
P_{D}=P_{T}\left(\frac{c}{4 \pi f r_{D}}\right)^{n_{1}}
$$

а отраженного -

$$
P_{R T}=\gamma P_{T}\left(\frac{c}{4 \pi f\left(r_{D}+c T\right)}\right)^{n_{2}}
$$

(где $\gamma$ - отраженная доля мощности, $f-$ средняя частота сигнала, $c$ - скорость света, $r_{D}-$ длина пути прямого луча, $c T-$ набег лучей, которые попадут на текущую позицию хаотического радиоимпульса через время $T$ после предшествующего), из отношения $P_{R T} / P_{D}$ можно получить предельное значение мощности $P_{R T}^{*}$ для отраженных лучей в приемнике по прошествии времени $T$ после прихода прямого луча. Для $n_{2}=2 n_{1}$ имеем

$$
P_{R T}^{*}=\gamma P_{D}\left(\frac{c}{4 \pi f r_{D}}\right)^{n_{1}}\left(\frac{1}{1+c T / r_{D}}\right)^{2 n_{1}}
$$

Для $n_{1}$ в (3) можно принять значение $n_{1}=1.6$, что согласуется с другими экспериментальными данными по измерению параметров СШП-каналов [1]. Значение $\gamma$ можно положить равным $\gamma=1$ (полное отражение), а для отношения $c T / r_{D}$ считать, что $c T / r_{D} \ll 1$ (наихудший случай).

В итоге обнаружение прямого луча на фоне отраженного должно осуществляться с пороговым значением $H>P_{R T}^{*}$. Здесь значение $P_{R T}^{*}(3)$ вычисляется на основе усредненных значений амплитуд огибающей хаотических радиоимпульсов по прямому и отраженному лучам. $\mathrm{B}$ то же время значения амплитуд огибающей последовательности хаотических радиоимпульсов варьируются от импульса к импульсу, подчиняясь некоторому распределению. Поэтому для предотвращения ошибок, вызванных разбросом в мощности поступающих импульсов, необходимо также учесть ширину этих распределений. На рис. 3 приведены экспериментальные распределения амплитуд огибающих импульсов прямого и отраженного лучей. Распределения ограничены: разброс амплитуд находится в пределах $\pm 0.1 \mathrm{~V}$ от их средних значений. Это значит, что обнаружение прямого луча на фоне отраженного можно осуществлять с пороговым значением мощности $H>P_{R T}^{*}+\Delta P_{R T}$, где $\Delta P_{R T}=0.1 / \alpha$.

В результате появляется физическая возможность устранить влияние межимпульсной интерференции и достигнуть характеристик обнаружения, близких по вероятности ошибки к характеристикам канала с одним прямым лучом, без реализации процедуры выделения отдельных лучей, осуществляемой в RAKE-приемнике.

\section{Конфликт интересов}

Авторы заявляют, что у них нет конфликта.

\section{Список литературы}

[1] Molisch A.F. // Proc. of the IEEE. 2009. V. 97. N 2. P. $353-$ 371.

[2] Andreyev Yu.V., Dmitriev A.S., Efremova E.V., Khilinsky A.D., Kuzmin L.V. // Int. J. Bifurcation Chaos. 2005. V. 15. N 11. P. 3639-3651.

[3] Кузьмин Л.В. // Радиотехника и электроника. 2011. Т. 56. № 4. C. 399-416.

[4] Дмитриев А.С., Рыжков А.И., Попов М.Г. // Радиоэлектроника. Наносистемы. Информационные технологии. 2018. T. 10. № 2. C. 313-322.

[5] Кузьмин Л.В., Морозов В.А. // Радиотехника и электроника. 2009. Т. 54. № 3. С. 329-338.

[6] Кузьмин Л.В., Гриневич А.В., Ушаков М.Д. // Письма в ЖТФ. 2018. Т. 44. В. 16. C. 48-56.

[7] Witrisal K., Leus G., Janssen G.J.M., Pausini M., Troesch F., Zasowski T., Romme J. // IEEE Signal Proc. Mag. 2009. V. 26. N 4. P. 48-66.

[8] Ma H., Wang X., Lu Y., Tian L. // IEEE Int. Conf. on smart grid and smart cities (ICSGSC). Singapore, 2017. P. 308-311.

[9] Arias-de-Reyna E., Murillo-Fuentes J.J., Boloix-Tortosa R. // IEEE Signal Proc. Lett. 2015. V. 22. N 11. P. 2019-2023.

[10] Shen B., Yang R., Cui T., Kwak K. // IEEE Int. Conf. on ultra-wideband. Nanjing, 2010. P. 1-4.

[11] $W u W$. // IEEE Int. Conf. on ultra-wideband. Singapore, 2007. P. 556-561.

[12] Dos Santos A.F., Rave W., Fettweis G. // IEEE Int. Conf. on ultra-wideband. Hannover, 2008. P. 41-44.

[13] Cassioli D., Win M.Z., Vatalaro F., Molisch A.F. // IEEE Trans. Wireless Commun. 2007. V. 6. N 4. P. 1265-1275.

[14] Benotmane N.B., Elahmar S.A., Dayoub I., Hamouda W. // IEEE Trans. Vehicular Technol. 2018. V. 67. N 8. P. 7749 7753.

[15] Analog Devices. Data Sheet $1 \mathrm{MHz}$ to $4 \mathrm{GHz}, 80 \mathrm{~dB}$, Logarithmic Detector/Controller; http://www.analog.com/media/en/technical-documentation/data-sheets/ADL5513.pdf

\section{Финансирование работы}

Работа выполнена по государственному заданию. 